\title{
Artigo/Article
}

\section{Características epidemiológicas e clínicas das reações hansênicas em indivíduos paucibacilares e multibacilares, atendidos em dois centros de referência para hanseníase, na Cidade de Recife, Estado de Pernambuco}

\author{
Characteristics of leprosy reactions in paucibacillary and multibacillary individuals attended \\ at two reference centers in Recife, Pernambuco
}

\author{
Márcia Almeida Galvão Teixeiraa ${ }^{1}$, Vera Magalhães da Silveira ${ }^{2}$ e Emmanuel Rodrigues de França ${ }^{3}$
}

\begin{abstract}
RESUMO
Introdução: As reações são frequentes e importantes no contexto da hanseníase, representando uma significativa parcela de pacientes com incapacidades e submetidos ao retratamento da hanseníase. A caracterização clínico-epidemiológica dos padrões reacionais é primordial para o manejo dos pacientes. O objetivo desse trabalho é descrever as características epidemiológicas e clínicas das reações hansênicas em indivíduos paucibacilares e multibacilares. Métodos: Estudo transversal onde foram avaliados 201 pacientes com história de quadro reacional, atendidos em dois centros de referência para tratamento da hanseníase. Variáveis como baciloscopia inicial, sexo, idade, fototipo, procedência, forma clínica, tipo de tratamento e de reação, índice baciloscópico final e período de surgimento da reação em relação ao tratamento foram avaliados. A análise estatística foi realizada usando-se frequências simples. Para cálculo dos fatores de risco para as formas multibacilares, foram realizadas análises univariada e multivariada. Resultados: Sexo masculino, idade entre 30-44 anos, fototipo V, a forma clínica borderline, tratamento regular, reação tipo I, neurite, presença de 10 a 20 nódulos e surgimento da reação hansênica durante o tratamento foram os achados mais frequentes. Conclusões: Predominaram os indivíduos do sexo masculino que se associaram a um maior risco de desenvolvimento da forma multibacilar. As reações hansênicas foram mais frequentes durante o tratamento, os pacientes multibacilares foram mais propensos ao retratamento da hanseníase e aqueles com reações tipo I e II, apresentaram maior frequência de neurite, linfadenopatia, artrite e irite do que aqueles com reação isolada.

Palavras-chaves: Hanseníase. Reações hansênicas. Epidemiologia. Sinais e sintomas.
\end{abstract}

\begin{abstract}
Introduction: Significant reactions frequently occur among leprosy cases, and thus a significant proportion of leprosy patients present disabilities and undergo leprosy retreatment. Clinicalepidemiological characterization of reaction patterns is essential for managing such patients. Objective to describe the epidemiological and clinical characteristics of leprosy reactions among paucibacillary and multibacillary individuals. Methods: In this cross-sectional study, 201 patients with histories of reactions who were attended at two reference centers for leprosy treatment were evaluated. Variables such as initial bacilloscopy, sex, age, skin phototype, origin, clinical presentation, type of treatment, type of reaction, final bacilloscopy index and time of reaction onset in relation to the treatment were evaluated. Statistical analysis was performed using simple frequencies. To calculate risk factors for multibacillary forms, univariate and multivariate analyses were performed. Results: Male sex, age between 30 and 44 years, phototype V, borderline clinical form, regular treatment, type I reaction, neuritis, presence of 10 to 20 nodules and onset of the leprosy reaction during the treatment were the most frequent findings. Conclusions: Male patients predominated and were associated with greater risk of developing the multibacillary forms. Leprosy reactions occurred most frequently during the treatment. Multibacillary patients were more likely to need leprosy retreatment, and those with type I and type II reactions presented greater frequency of neuritis, lymphadenopathy, arthritis and iritis than did those with isolated reactions.
\end{abstract}

Key-words: Leprosy. Leprosy reactions. Epidemiology. Signs and symptoms.

1. Departamento de Dermatologia, Universidade de Pernambuco, Recife, PE. 2. Departamento de Medicina Tropical, Universidade Federal de Pernambuco, Recife, PE. 3. Departamento de Dermatologia, Universidade de Pernambuco, Recife, PE.

Endereço para correspondência: Dra Márcia Almeida Galvão Teixeira. Rua Jornalista Guerra de Holanda, 158/1102 Casa Forte. 52061010 Recife, PE.

Tel: 5581 3427-2268/9245-4349.

e-mail: marciateixeira@folha.rec.br

Recebido para publicação em 13/10/2009

Aceito em 25/03/2010

\section{INTRODUÇÃO}

Os indivíduos hansenianos podem ser surpreendidos por quadros ou estados reacionais, intercorrências no curso da doença, que estão presentes em cerca de 10 a 50\% dos casos, principalmente, nas formas multibacilares e constituem importantes fatores de risco para retratamento da hanseníase, além de responsáveis por abandono de tratamento e pelas incapacidades ${ }^{1,2}$.

Brito $^{3}$, em 2004, estudando 310 pacientes hansenianos, observou que aqueles que sofriam com episódios reacionais após alta, apresentavam três vezes mais possibilidades de retratamento por suspeita de recidiva da hanseníase que o grupo controle.

As reações hansênicas refletem fenômeno de hipersensibilidade aguda diante dos antígenos do Mycobacterium leprae 4 e decorrem de processo imunológico acompanhado de aumento de citocinas pró-inflamatórias, principalmente IFN- $\gamma$, IL-12, IL1, IL-2, IL-4, IL-6, IL-8, IL-10, entre outras ${ }^{5,6}$, além de imunocomplexos ${ }^{7}$.

Os quadros reacionais podem surgir antes ou, mais frequentemente, durante ou após o tratamento. A duração e o número desses surtos reacionais dependem muitas vezes da forma clínica, bem como do índice baciloscópico inicial ${ }^{8,9}$.

As reações hansênicas são classificadas em dois tipos de acordo com Jopling ${ }^{10}$ : reação hansênica tipo I ou reversa (RR), quando está envolvida a imunidade celular, e a reação hansênica tipo II com os tipos eritema nodoso hansênico $(\mathrm{ENH})$, eritema polimorfo (EP) e eritema nodoso necrotizante (ENN), com participação mais efetiva da imunidade humoral. A neurite pura ou isolada pode ser classificada como reação tipo I ou um terceiro tipo de reação hansênica. A reação tipo I pode ser ascendente, quando há aumento da imunidade específica ou descendente quando há uma queda da imunidade e a expressão clínica é semelhante ${ }^{11}$. 
Episódios de reação tipo I ou reversa acometem entre 10 e $33 \%$ dos pacientes com hanseníase e surgem, geralmente durante o tratamento ou após o primeiro ano da alta e apresentam as seguintes características clínicas: infiltração de lesões antigas associada ao surgimento de novas lesões em forma de manchas ou placas infiltradas, eritema, dor, lesões vésico-bolhosas, ulcerações, hiperestesia, parestesia, mal estar, dor ou espessamento de nervos periféricos com perda da função sensitivomotora e, mais raramente, febre, déficit da função neural na ausência de sintomas (neuropatia silenciosa), acometendo principalmente os nervos ulnar e tibial posterior ${ }^{1,12}$

As reações tipo II são caracterizadas pelo surgimento abrupto de nódulos que podem variar de poucos a inúmeros, de coloração rósea, que podem evoluir para necrose, nas formas mais graves do ENH. Também, vem acompanhadas de sintomatologia relacionada ao acometimento ocular, hepático, esplênico, de linfonodos, peritônio, testículos, articulações, tendões, músculos, ossos e rins. Pode haver febre, leucocitose e, geralmente apresentam-se em múltiplos episódios ${ }^{13,14}$.

O uso de corticóides no tratamento da reação reversa e da talidomida para o tratamento do eritema nodoso é recomendado pela organização mundial de saúde ${ }^{15,16}$.

As reações hansênicas constituem intercorrências na doença, com sinais e sintomas que levam o paciente ao sofrimento e sequelas neurológicas, muitas vezes mais expressivas que as esperadas na hanseníase sem quadro reacional. Elas representam fenômenos imunológicos, frequentemente pouco entendidos que refletem em quadro clínico peculiar exigindo a atenção do dermatologista.

Este trabalho objetivou a descrição das características clínicas de pacientes com hanseníase reacional atendidos em dois centros de referência.

\section{MÉTODOS}

Por meio de um estudo transversal, foram investigados 201 pacientes com história de quadro reacional hansênico, atendidos nos ambulatórios de hanseníase em duas unidades de referência (Centro Integrado de Saúde Amaury de Medeiros e Policlínica Lessa de Andrade) da Cidade do Recife-PE, no período de março de 2007 a dezembro de 2008.

Para classificação dos pacientes segundo forma baciloscópica, adotaram-se os critérios da $\mathrm{OMS}^{16}$, que consistem na pesquisa de BAAR em material linfo cutâneo e na apresentação clínica. Foram classificados como paucibacilares, os pacientes hansenianos com: baciloscopia inicial negativa, menos de cinco lesões e até um tronco nervoso afetado. Os pacientes multibacilares foram selecionados entre os que apresentavam baciloscopia inicial positiva. Indivíduos com a forma neurítica pura de hanseníase não participaram do estudo.

Considerou-se reação reversa, ou tipo I, a que apresentasse pelo menos uma das seguintes características: infiltração em lesões antigas de hanseníase acompanhadas de edema e dor, surgimento de novas lesões em forma de placas eritemato-infiltradas, tricofitóides com distúrbios de sensibilidade, ou espessamento em um ou mais nervos periféricos associado a quadro doloroso. Essas características poderiam estar associadas a outros achados, como febre, mal estar, hipersensibilidade palmar e plantar, lesões vésico-bolhosas, ulceração e edema em mãos e pés ${ }^{12}$.

Foi caracterizada como reação tipo II, ou eritema nodoso hansenótico, aquela que apresentasse nódulos eritematosos, dolorosos espontaneamente ou à palpação, isolados ou associados a qualquer uma das manifestações: febre, mal estar, astenia, vesículas / bolhas ou úlceras, espessamento doloroso em um ou mais nervos periféricos, linfadenopatia, irite, artrite ou artralgia e edema ou dor em mãos e pés ${ }^{12}$.

A reação tipo II foi quantificada pela contagem do número de nódulos nos seguimentos corporais afetados: membros inferiores e abdome ou, membros superiores, tórax e face e foi considerada leve, na presença de menos de 10 nódulos, moderada quando havia 10 a 20 nódulos e severa, em presença de mais de 20 nódulos por seguimento corporal afetado.

Considerou-se que o paciente com reação realizou tratamento regular para a hanseníase quando não houve abandono por mais de três meses consecutivos da poliquimioterapia e tratamento irregular quando houve abandono do tratamento por mais de três meses.

O esquema terapêutico de hanseníase foi considerado padrão, quando foram administradas as drogas preconizadas pela OMS, ou seja, dapsona, clofazimina (exceto para os paucibacilares) e rifampicina durante seis meses para as formas paucibacilares e um ano para as formas multibacilares. Classificou-se o esquema como alternativo na ocorrência do uso de outras drogas ou de tempo de tratamento diferente.

A coleta de dados constou de entrevista, anamnese, exame físico, consulta ao prontuário e preenchimento de protocolo específico. Descreveram-se as variáveis relativas a fototipo segundo Fitzpatrick ${ }^{17}$ (tipo I, II, III, IV, V e VI), procedência (Recife, região metropolitana e interior) e forma clínica (tuberculóide (TT), borderline (BT, BB e $\mathrm{BL}$ ) e lepromatosa (LL)). Compararam-se as características clínicas entre os pacientes reacionais avaliando: idade, sexo, baciloscopia inicial e final, tipo de tratamento (convencional ou alternativo), tipo de reação (I ou II), aspectos clínicos da reação, tempo de surgimento da reação em relação ao início do tratamento e drogas utilizadas.

Com o objetivo de identificar os principais fatores de risco para o indivíduo ser multibacilar, e consequentemente apresentar surtos reacionais mais graves e numerosos, fez-se uma análise univariada, comparando a proporção dessa forma baciloscópica nas diferentes categorias pelo teste qui-quadrado de Pearson ${ }^{18,19}$.

Para análise multivariada, optou-se pela técnica de regressão $\operatorname{logística~}^{18}$, incluindo as variáveis que apresentaram nível de significância menor que 0,20 , na análise univariada. A seleção das variáveis foi feita através do método stepwise forward, estabelecendose nível de significância de 0,05 para entrada de variáveis e de 0,10 para saída. As variáveis selecionadas tiveram suas interações analisadas em uma matriz de correlação, sendo incorporadas ao modelo todas as interações com coeficiente de correlação $\geq 0,5$.

$\mathrm{O}$ gerenciamento do banco de dados foi realizado com o programa MS Office Excel versão 2003; a análise estatística, com o programa Statistical Package for Social Sciences (SPSS ${ }^{\circ}$ for Windows versão 12.0, e a confecção de gráficos e tabelas com o MS Office Word" e Excel" versão 2003.

Os pacientes que participaram do estudo assinaram o Termo de Consentimento Livre Esclarecido e foram informados dos riscos e benefícios além da confidencialidade do estudo.

\section{Ética}

O projeto teve a aprovação do Comitê de Ética do Centro Integrado de Saúde Amaury de Medeiros, onde foi registrada sob $n^{\circ}$.015/07. 


\section{RESULTADOS}

Quanto às variáveis de caracterização dos pacientes, foi possível identificar um predomínio do sexo masculino e da faixa etária entre $30 \mathrm{e}$ 44 anos (Tabela 1). Houve maior frequência de pacientes com fototipo V (74; 36,8\%) e procedentes da Cidade do Recife (129; 64,2\%).

A Tabela 1 mostra que dos 201 indivíduos estudados, 100 foram classificados como paucibacilares e 101 como multibacilares, dentre os quais constatou se um predomínio de baciloscopia menor que três $(73 ; 72,3 \%)$.

TABELA 1 - Distribuição das características demográficas e clínicas dos 201 pacientes reacionais atendidos no CISAM e Centro de Saúde Lessa de Andrade.

\begin{tabular}{|c|c|c|c|c|c|c|c|c|}
\hline \multirow[b]{3}{*}{ Variáveis } & \multirow{2}{*}{\multicolumn{2}{|c|}{$\begin{array}{c}\text { Número } \\
\text { de pacientes }\end{array}$}} & \multicolumn{6}{|c|}{ Reação } \\
\hline & & & \multicolumn{2}{|c|}{ Tipo I } & \multicolumn{2}{|c|}{ Tipo II } & \multicolumn{2}{|c|}{ Tipo I e II } \\
\hline & $\mathrm{n}^{\mathrm{o}}$ & $\%$ & $\mathrm{n}^{\mathrm{o}}$ & $\%$ & $\mathrm{n}^{\mathrm{o}}$ & $\%$ & $\mathrm{n}^{\mathrm{o}}$ & $\%$ \\
\hline \multicolumn{9}{|l|}{ Sexo } \\
\hline masculino & 131 & 65,2 & 99 & 65,6 & 21 & 67,7 & 11 & 57,9 \\
\hline feminino & 70 & 34,8 & 52 & 34,4 & 10 & 32,3 & 8 & 42,1 \\
\hline \multicolumn{9}{|l|}{ Idade (anos) } \\
\hline $0-14$ & 8 & 4,0 & 6 & 4,0 & 0 & 0,0 & 2 & 10,5 \\
\hline $15-29$ & 40 & 19,9 & 31 & 20,5 & 9 & 29,0 & 0 & 0,0 \\
\hline $30-44$ & 69 & 34,3 & 46 & 30,5 & 14 & 45,2 & 9 & 47,4 \\
\hline $45-59$ & 61 & 30,4 & 46 & 30,5 & 7 & 22,6 & 8 & 42,1 \\
\hline$\geq 60$ & 23 & 11,4 & 22 & 14,5 & 1 & 3,2 & 0 & 0,0 \\
\hline \multicolumn{9}{|l|}{ Tipo baciloscopia } \\
\hline paucibacilar & 100 & 49,8 & 100 & 66,2 & 0 & 0,0 & 0 & 0,0 \\
\hline multibacilar & 101 & 50,2 & 51 & 33,8 & 31 & 91,2 & & 00,0 \\
\hline $0,09-2,99^{1}$ & 73 & 72,3 & 49 & 33,1 & 11 & 32,4 & 13 & 68,4 \\
\hline$\geq 3,00$ & 28 & 27,7 & 2 & 1,4 & 20 & 58,8 & 6 & 31,6 \\
\hline \multicolumn{9}{|l|}{ Baciloscopia final } \\
\hline negativa & 56 & 27,9 & 52 & 34,4 & 2 & 6,5 & 2 & 10,5 \\
\hline positiva & 24 & 11,9 & 7 & 4,7 & 11 & 35,5 & 6 & 31,6 \\
\hline não realizada & 121 & 60,2 & 92 & 60,9 & 18 & 58,1 & 11 & 57,9 \\
\hline \multicolumn{9}{|c|}{ Início da reação hansênica } \\
\hline no tratamento & 136 & 67,7 & 99 & 65,6 & 21 & 67,7 & 16 & 84,2 \\
\hline$<6$ meses da alta & 38 & 18,9 & 28 & 18,5 & 8 & 25,8 & 2 & 10,5 \\
\hline 6-12 meses da alta & 9 & 4,5 & 6 & 4,0 & 2 & 6,5 & 1 & 5,3 \\
\hline$>1$ ano da alta & 18 & 8,9 & 18 & 11,9 & 0 & 0,0 & 0 & 0,0 \\
\hline \multicolumn{9}{|c|}{ Drogas usadas na reação } \\
\hline prednisona & 152 & 75,6 & 140 & 92,7 & 7 & 22,6 & 5 & 26,3 \\
\hline talidomida & 6 & 3,0 & 0 & 0,0 & 6 & 19,4 & 0 & 0,0 \\
\hline ambas & 39 & 19,4 & 7 & 4,6 & 18 & 58,1 & 14 & 73,7 \\
\hline outras $^{2}$ & 4 & 2,0 & 4 & 2,7 & 0 & 0,0 & 0 & 0,0 \\
\hline
\end{tabular}

${ }^{1}$ Número de bacilos identificados em pacientes multibacilares - Teste qui quadrado de Pearson $-p<0,001$.

${ }^{2}$ Outras drogas para tratamento de quadros reacionais incluíram carbamazepina, clofazimina, e pentoxiflina.

A reação hansênica foi classificada como tipo I em $151(75,1 \%)$ pacientes, como tipo II em $31(15,4 \%)$ e reação tipo I e tipo II em $19(9,5 \%)$ pacientes. Em todos os casos, o início da reação hansênica foi mais frequente durante o tratamento $(67,7 \%)$ ou num período menor que seis meses, após a alta medicamentosa. Para o tratamento da reação nesses pacientes, a droga de escolha foi a prednisona, isolada $(75,6 \%)$ ou associada $(19,4 \%)$ à talidomida (Tabela 1). Dos pacientes estudados, a maioria submeteu-se ao tratamento padrão (87\%-175/201) e regular (90\% - 180/201) da hanseníase.

Quanto à distribuição dos pacientes, segundo a reação hansênica, expressa na Tabela 1, identificou-se que as do tipo I ocorreram mais frequentemente nos pacientes na faixa etária de 30 a 59 anos, paucibacilares ou multibacilares com contagem de bacilos menor que 3,00 e com início durante o tratamento da hanseníase ou após um ano do término da alta medicamentosa. As reações do tipo II caracterizaram-se por predominarem em pacientes na faixa etária de 30 a 44 anos, multibacilares e com contagem de bacilos igual ou maior que 3,00 .

Houve associação significante entre baciloscopia menor que 3,00 e reação tipo I e baciloscopia igual ou maior que 3,00 e a reação tipo II $(\mathrm{p}<0,001)$.

Em relação às formas clínicas, dos 201 indivíduos estudados, 40 (19,9\%) foram tuberculóides, 118 (58,7\%) foram borderline e 43 $(21,4 \%)$ Virchowiana.

As características clínicas dos quadros reacionais encontram-se nas Figuras 1 e 2. Quanto às características comuns às reações tipo I e II, constatou-se que a neurite foi o comprometimento mais frequente entre os tipos reacionais (Figura 1). Dentre os 151 pacientes com reação tipo I, a neurite ocorreu de forma isolada em 34 (22,5\%) deles e as lesões em placa, exclusivas desse grupo, apresentaram-se como comprometimento unicamente cutâneo em 46 (30,5\%) pacientes.

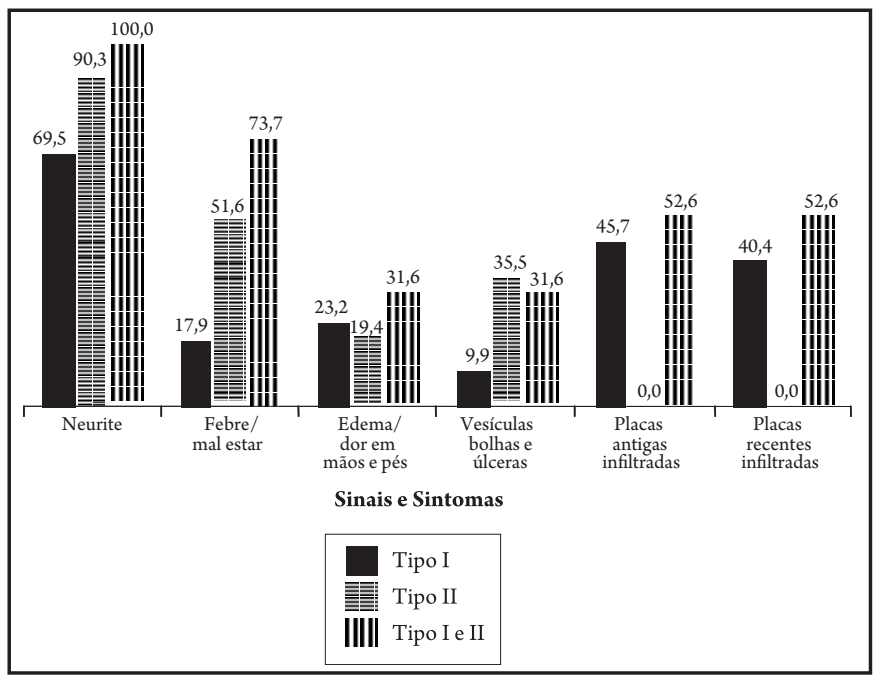

FIGURA 1 - Distribuição dos achados clínicos comuns às reações tipo I e II, diagnosticadas em 201 pacientes segundo reação hansênica acompanhados em dois Centros de Referência em Recife, PE.

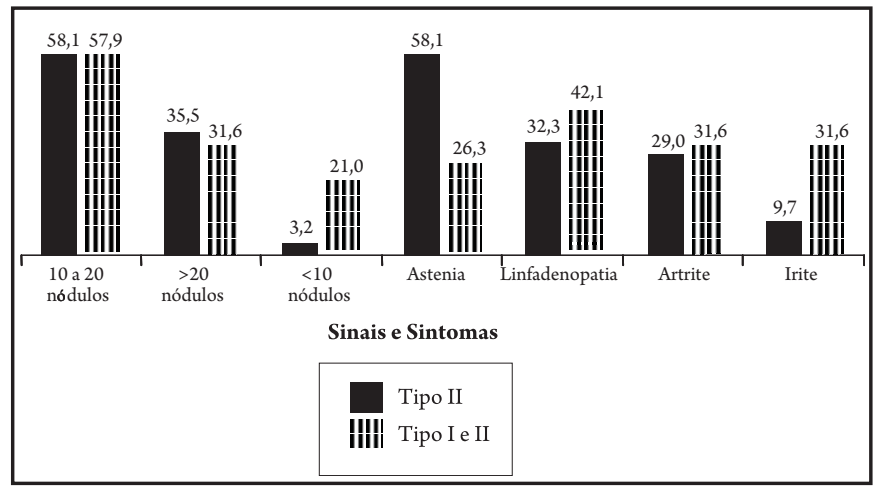

FIGURA 2 - Distribuição dos achados clínicos dos pacientes com reação hansênica tipo II, isolada ou associada à reação tipo I, acompanhados em dois centros de referência em Recife, $P E$. 
Na Figura 2, apresentam-se as manifestações clínicas de 50 pacientes com reação hansênica tipo II, observando-se que no grupo com ambas as reações, a presença de menos de 10 nódulos por segmento corporal afetado, linfadenopatia, artrite e irite foram mais frequentes do que no grupo com reação tipo II isolada.

Para determinar a associação entre as variáveis idade, sexo, início da reação hansênica, acometimento neural e retratamento da hanseníase com a forma multibacilar, procedeu-se à análise univariada (Tabela 2). Determinou-se que pacientes submetidos ao retratamento tinham chance aproximadamente uma vez maior de serem multibacilares. A idade, sexo, época de início da reação e acometimento neural não se associaram à baciloscopia inicial.

Constatou-se também que a distribuição das reações hansênicas, associou-se à baciloscopia, sendo mais frequente a reação tipo I em paucibacilares e tipo II em multibacilares $(p<0,001)$.

As variáveis sexo, início da reação e retratamento foram incluídas na análise multivariada e submetidas à regressão logística, por apresentarem valor de p menor que $20 \%$, cujos resultados estão apresentados na Tabela 3. Observou-se que pacientes do sexo masculino tinham chance 0,81 vezes maior de serem multibacilares quando comparados ao feminino.

TABELA 2 - Análise univariada da associação entre variáveis de risco e a forma multibacilar em 201 indivíduos com quadro reacional.

\begin{tabular}{|c|c|c|c|c|c|}
\hline \multirow[b]{2}{*}{ Variáveis } & \multicolumn{4}{|c|}{ Multibacilar } & \multirow[b]{2}{*}{$p$} \\
\hline & $\mathrm{N}^{1}$ & $\mathrm{n}^{\mathrm{o}}$ & $\%$ & OR (I.C.95\%) & \\
\hline \multicolumn{6}{|l|}{ Idade (anos) } \\
\hline$\leq 44$ & 117 & 62 & 53,0 & 1,0 & \\
\hline$>44$ & 84 & 39 & 46,4 & $0,77(0,44-1,35)$ & 0,359 \\
\hline \multicolumn{6}{|l|}{ Sexo } \\
\hline feminino & 70 & 29 & 41,4 & 1,0 & \\
\hline masculino & 131 & 72 & 55,0 & $1,73(0,96-3,10)$ & 0,068 \\
\hline \multicolumn{6}{|l|}{ Início da reação } \\
\hline no tratamento ou $<6$ meses da alta & 174 & 91 & 52,3 & 1,0 & \\
\hline$\geq 6$ meses da alta & 27 & 10 & 37,0 & $0,54(0,23-1,24)$ & 0,140 \\
\hline \multicolumn{6}{|l|}{ Acometimento neural } \\
\hline não & 61 & 28 & 45,9 & 1,0 & \\
\hline $\operatorname{sim}$ & 140 & 73 & 52,1 & $1,28(0,70 ; 2,35)$ & 0,416 \\
\hline \multicolumn{6}{|l|}{ Retratamento } \\
\hline não & 152 & 70 & 46,1 & 1,0 & \\
\hline $\operatorname{sim}$ & 49 & 31 & 63,3 & $2,02(1,04 ; 3,91)$ & $0,036^{2}$ \\
\hline
\end{tabular}

${ }^{1}$ base de cálculo percentual: - 201 pacientes, ${ }^{2}$ valor de $p$ significante.

TABELA 3 - Regressão logística da variável sexo com a forma multibacilar em 201 indivíduos reacionais.

\begin{tabular}{lcccc}
\hline Variável independente & Coeficiente & $p$ & Odds Ratio & IC 95\% \\
\hline Sexo (masculino x feminino) & 0,59 & 0,049 & 1,81 & $1,01-3,43$ \\
Constante & $-0,72$ & 0,008 & & \\
\hline
\end{tabular}

\section{DISCUSSÃo}

A identificação e o manejo dos pacientes com episódios reacionais hansênicos constituem um desafio para o clínico, porque esses episódios requerem diagnóstico precoce, já que as sequelas são graves. Quando os quadros reacionais são tardios, após a alta medicamentosa, aumenta a dificuldade em diagnosticar devido à necessidade de diferenciação com os quadros de recidiva. $\mathrm{O}$ retardo no diagnóstico diferencial entre recidiva e reação hansênica acarretará atraso também na conduta terapêutica, o que contraria o atendimento das necessidades dos pacientes por intervenções rápidas.

A caracterização clínica dos pacientes reacionais pode contribuir positivamente nesse sentido, do que deriva a importância dos resultados aqui apresentados, já que os estudos sobre reação hansênica em população nordestina são escassos.

Em relação ao sexo dos pacientes, observou-se maior frequência de quadros reacionais em homens, numa proporção de 1,87:1,00 homens:mulheres, contrariando a distribuição populacional da Cidade do Recife, que se igualava a 0,8:1,00, estatísticas de 2007. Considerando que a expectativa de vida da mulher, desde a adolescência, é maior que a do homem, a maior proporção de homens reacionais hansênicos pode ser uma característica da doença ${ }^{20}$.

A proporção do presente estudo foi menor que a de 3,75:1,00, identificada em pesquisa de reações hansênicas entre pacientes tailandeses ${ }^{11}$. Porém, maior que a de 1,18:1,00, constatada entre 290 pacientes, estudados no Rio de Janeiro ${ }^{21}$.

No presente estudo, ao incluir a variável sexo na análise multivariada (Tabelas 2 e 3), para determinação dos fatores de risco para formas multibacilares, e consequentemente, para predisposição aos quadros reacionais mais graves, comprovou-se que indivíduos do sexo masculino tinham chance 0,81 vezes maior de serem multibacilares. Esse fato aponta para a necessidade de um acompanhamento mais cuidadoso dos pacientes do sexo masculino, por apresentarem maior propensão às formas mais graves de reação.

Quanto à distribuição etária, apesar da maior frequência da reação tipo I numa faixa mais ampla (entre 30 e 59 anos) que aquela de pacientes com reação tipo II (entre 30 e 44 anos) (Tabela 1), essa variável não se associou a maior risco de desenvolvimento da forma multibacilar. Mesmo assim, dois aspectos dessa distribuição etária são relevantes. Em primeiro lugar, deve-se considerar que o adoecimento do homem na idade entre 30 e 49 anos exerce impacto psicossocial, devido à maior atividade laborativa nessa faixa etária. Em segundo lugar, é preocupante o achado de crianças menores de 15 anos de idade com reação hansênica, principalmente pela consideração de que, nessa faixa etária, devem ter maior convívio com membros familiares, indicando que sua contaminação foi domiciliar.

O predomínio do fototipo $\mathrm{V}$ e da procedência da Cidade do Recife, entre os pacientes (Tabela 1), pareceu refletir tão somente a cor da pele de habitantes da cidade e a situação geográfica dos centros de referência, que foram escolhidos por locais da presente pesquisa.

Do ponto de vista clínico, a associação da forma multibacilar com contagem de BAAR igual ou maior que 3,00 e desenvolvimento de reação tipo II isolada $(58,8 \%)$ esteve em consonância com a literatura ${ }^{16,22}$.

O predomínio de pacientes multibacilares com baciloscopia menor que 3,00 (72,3\%; Tabela 1 ) pode induzir ao raciocínio equivocado de que essa associação indicaria melhor prognóstico, pela menor carga bacilar. No entanto, quando se observa a frequência de ambos os quadros reacionais nos multibacilares, os quais apresentam um quadro clínico mais grave com sinais e sintomas incapacitantes como neurite, edema e dor em mãos e pés, bolhas e úlceras, linfadenopatia e artrite (Figuras 1 e 2), este foi mais frequente $(21,4 \%-6 / 28)$, nos multibacilares com baciloscopia igual ou maior que 3,00 . 
A baciloscopia, tanto inicial, quanto final, é um exame que integra o arsenal diagnóstico da hanseníase e é acessível, mesmo em países de baixa renda e em desenvolvimento. A pesquisa de BAAR pode auxiliar no direcionamento do diagnóstico diferencial entre recidivas e desencadeamento de reação hansênica.

A não realização da baciloscopia após alta medicamentosa em alguns dos pacientes deste estudo pareceu um reflexo negativo das recomendações do Ministério da Saúde quanto ao manejo do paciente em tratamento em esquema de poliquimioterapia para hanseníase. Se, ao diagnóstico, a baciloscopia é um critério essencial, o mesmo deveria ocorrer após alta, pois auxilia na diferenciação entre as reações hansênicas, reconhecidas como eventos graves nos quais este exame não é modificado, e as recidivas da hanseníase que revelam modificação na baciloscopia após alta medicamentosa, em se tratando de indivíduos multibacilares.

Deve-se ressaltar que a redução do índice baciloscópico diminui as chances de reação ${ }^{15}$. Ainda que se lançasse mão do exame histopatológico, uma importante ferramenta para diferenciar a reação tipo I da recidiva, seus resultados não substituem a baciloscopia para estimativa de risco de desenvolvimento da reação hansênica. $\mathrm{O}$ exame histopatológico comprova o tipo de reação e a distingue da recidiva, porém muitas vezes não está disponível para rotina, mesmo nos centros de referência para hanseníase ${ }^{23}$.

Um fato relevante, neste trabalho, é que o primeiro episódio de reação hansênica, tanto tipo I como tipo II, surgiu na maioria $(86,6 \%)$ dos indivíduos ainda durante o tratamento ou após seis meses da alta. A presença de estados reacionais durante a terapêutica parece ser reflexo do comportamento imunológico do indivíduo e reforça a teoria de que o próprio tratamento para a hanseníase pode ser um fator precipitante para o surgimento do quadro reacional. Em pacientes com grande carga bacilar, a presença contínua de antígenos de bacilos mortos aumenta o risco de reações hansênicas já que esta eliminação bacilar é lenta e depende do sistema fagocitário do indivíduo na ordem de 0,6 a 1,0 log/ano. Esse mecanismo também explica porque as reações podem ocorrer logo após alta medicamentosa ${ }^{24,25}$.

No presente estudo, o surgimento da reação tipo II ainda durante o tratamento pareceu contrariar os achados de uma grande coorte ${ }^{26}$, de que essa reação é mais frequente no primeiro ou segundo ano após alta da terapia multidroga. O fato de clofazimina ter sido utilizada durante o tratamento e ter efeito antiinflamatório poderia justificar o surgimento tardio do quadro reacional na forma lepromatosa.

O uso de talidomida em sete pacientes com a reação Tipo I (Tabela 1), pode refletir as dificuldades de condução diagnóstica e terapêutica que as reações hansênicas demandam, como por exemplo, a alternância dos tipos reacionais em multibacilares.

A constatação de que os pacientes multibacilares tiveram maior chance de necessitar retratamento (Tabela 2) trouxe informação adicional aos achados de um estudo tipo caso-controle, também realizado em população recifense ${ }^{3}$, no qual o retratamento foi três vezes mais freqüente nos indivíduos reacionais.

No presente trabalho, o predomínio da forma borderline foi um achado coerente com o critério de inclusão de pacientes reacionais, já que a característica dessa forma clínica é a instabilidade clínica e imunológica com a maior susceptibilidade aos quadros reacionais ${ }^{12}$.

Comparativamente, a reação tipo II é mais agressiva que a reação tipo I, mas essa observação só está adequada aos pacientes multibacilares, porque podem desenvolver ambas, isolada ou alternadamente ${ }^{13}$. Assim os pacientes paucibacilares, não sujeitos à reação tipo II, estiveram menos expostos a complicações, mas é importante ressaltar que tal achado não é tranquilizador, pois essas reações são acompanhadas de neurite que é responsável por danos e sequelas importantes ${ }^{1,9,13}$.

Concluímos que os pacientes reacionais desta amostra caracterizaram-se por predomínio do sexo masculino associado a um maior risco de desenvolvimento da forma multibacilar, apresentando predominantemente a forma clínica borderline, com desenvolvimento de reações hansênicas mais frequentes durante o tratamento. Os pacientes multibacilares foram mais propensos ao retratamento e aqueles com reações tipo I e II, apresentaram maior frequência de neurite, linfadenopatia, artrite e irite do que aqueles com reação isolada.

\section{AGRADECIMENTOS}

Aos pacientes que, num gesto de desprendimento, concordaram em participar do estudo e aos colegas dermatologistas e demais funcionários do CISAM e da Policlínica Lessa de Andrade.

\section{CONFLITO DE INTERESSE}

Os autores declaram não haver nenhum tipo de conflito de interesse no desenvolvimento do estudo.

\section{REFERÊNCIAS}

1. Andrade ARC, Lehman LF, Schreuder PAM. Como reconhecer e tratar reações hansênicas. Secretaria de Estado de Saúde de Minas Gerais. Belo Horizonte; 2005

2. Burns T, Breathnach S, Cox N, Griffiths C. editors Rook's Textbook. of Dermatology. $7^{\text {th }}$ ed, Oxford: Blackwell Sciences Ldt, Massachusetts 2004; p.29.1- 29.2.

3. Brito MFM. O retratamento em hanseníase: Identificação dos fatores de risco Um estudo caso-controle [tese]. Universidade Federal de Pernambuco. Recife; 2004

4. Mendonça VA, Costa RD, Melo GEBA, Antunes CM, Teixeira AL. Imunologia da hanseníase. An Bras Dermatol 2008; 83:343-350.

5. Goulart IMB, Penna GO, Cunha G. Imunopatologia da hanseníase: a complexidade da resposta imune do hospedeiro ao Mycobacterium leprae. Rev Soc Bras Med Trop 2002; 35:365-375.

6. Little D, Khanolkar-Young S, Coulthart A, Suneetha S, Lockwood DN Immunohistochemical analysis of cellular infiltrate and gamma interferon, interleukin-12, and inducible nitric oxide synthase expression in leprosy type 1 (reversal) reactions before and during prednisolone treatment. Infect Immun 2001; 69:3413-3417.

7. Ridley DS, Jopling WH. Classification of leprosy according to immunity: a fivegroup system. Int J Lepr Other Mycobact Dis 1966; 34:255-273.

8. Becx-Bleumink M, Berhe D. Occurrence of reactions, their diagnosis and management in leprosy patients treated with multidrug therapy; experience in the leprosy control program of the All Africa Leprosy and rehabilitation training center (ALERT) in Ethiopia. Int J Lepr Other Mycobact Dis 1992; 60:173184.

9. Kahawita IP, Walker SL, Lockwood DNJ. Leprosy type 1 reactions and erythema nodosum leprosum. An Bras Dermatol 2008; 83:75-82.

10. Jopling WH. Reactions in leprosy. Lepr Rev 1970; 41:62-63.

11. Scollard DM. Epidemiologic characteristics of leprosy reactions. Int J Lepr Other Mycobact 1994; 62: 559-569. 
12. Nery JAC, Sales AM, Illarramendi X, Duppre NC, Jardim MC, Machado AM. Contribuição ao diagnóstico e manejo dos estados reacionais. Uma abordagem prática. An Bras Dermatol 2006; 81:367-375.

13. Pfaltzgraff RE, Ramu G. Clinical leprosy. In: Hastings RC, editor. Leprosy. $2^{\text {nd }}$ ed. Edinburgh: Churchill Livingstone; 1994. p. 237-290.

14. Pocaterra L, Jain S, Reddy R, Muzaffarullah S, Torres O, Suneetha S, et al. Clinical course of erythema nodosum leprosum: an 11-year cohort study in Hyderabad, India. Am J Trop Med Hyg 2006; 74:868-879.

15. Guerra JG, Penna GO, de Castro LCM, Martelli CMT. Avaliação de série de casos de eritema nodoso hansênico: perfil clínico, base imunológica e tratamento instituído nos serviços de saúde. Rev Soc Bras Med Trop 2004; 37: 384-390.

16. World Health Organization [homepage on the Internet]. Global strategy for further reducing leprosy burden and sustaining leprosy control activities (20062010): Operational guidelines. [cited 2007 Dec 12]. Available from: http://www. who.int/lep/resources/SEAGLP20062.pdf<.

17. Fitzpatrick TB. Soleil et peau. J Med Esthet 1975; 2: 33-34.

18. Hosmer DW and Lemeshow, S. Applied Logistic Regression. New York: John Wiley \&Sons; 1989.

19. Vieira S. Introdução à Bioestatística. $3^{a}$ ed. Rio de Janeiro: Campus; 1998.

20. BlattJM. Considerações a cerca dos estados reacionais do portador de hanseníase no município de Itajaí. Saúde e Sociedade 1:2001.

21. Silva SF, Griep RH. Reação hansênica em pacientes portadores de hanseníase em centros de saúde da área de planejamento 3.2 do município do Rio de Janeiro. Han Intern 2007; 32:155-162

22. Saunderson P, Gebre S, Byass P. ENL reactions in the multibacillary cases of the AMFES cohort in central Ethiopia: incidence and risk factors. Lepr Rev 2000; 71:318-324.

23. Gallo MEN, Oliveira MLW. Recidivas e reinfecção em Hanseníase. Medicina, Ribeirão Preto 1997; 30:351-357.

24. Manandhar R, LeMaster JW, Roche PW. Risk factors for erythema nodosum leprosum. Int J Lepr Other Mycobact Dis 1999; 67:270-278.

25. Schreuder PAM. The occurrence of reactions and impairments in leprosy: experience in the leprosy control program of three provinces innortheastern Thailand, 1978-1995. II. Reactions. Int Jl of Lepr 1998; 66:159-169.

26. Kumar B, Dogra S, Kaur I. Epidemiological characteristics of leprosy reactions: 15 years experience from north India. Int $J$ Lepr Other Mycobact 2004; 72:125133. 